\title{
Saúde urinária e intestinal de profissionais de enfermagem em tempos de pandemia da COVID-19: uma reflexão teórica
}

\author{
Urinary and intestinal health of nursing professionals in pandemic times of COVID-19: a \\ theoretical reflection
}

Salud urinaria e intestinal de los profesionales de enfermería en tiempos de pandemia de COVID19: una reflexión teórica

\author{
Catarina de Melo Guedes \\ ORCID: https://orcid.org/0000-0003-2398-4527 \\ Coloplast do Brasil, Brasil \\ E-mail: catacatamg@hotmail.com \\ Norma Valéria Dantas de Oliveira Souza \\ ORCID: https://orcid.org/0000-0002-2936-3468 \\ Universidade Estadual do Rio de Janeiro, Brasil \\ E-mail: norval_souza@yahoo.com.br \\ Déborah Machado dos Santos \\ ORCID: https://orcid.org/0000-0002-1073-8223 \\ Universidade Federal do Rio de Janeiro, Brasil \\ E-mail: debuerj@yahoo.com.br \\ Patrícia Alves dos Santos Silva \\ ORCID: https://orcid.org/0000-0002-1482-0152 \\ Universidade Estadual do Rio de Janeiro, Brasil \\ E-mail: papatyenf@gmail.com \\ Manuela de Mendonça Figueiredo Coelho \\ ORCID: https://orcid.org/0000-0001-6182-9486 \\ Universidade Federal do Ceará, Brasil \\ E-mail: manumfc2003@yahoo.com.br
}

\begin{abstract}
Resumo
Objetivo: analisar os impactos da pandemia da COVID-19 na saúde urinária e intestinal dos profissionais de enfermagem. Método: estudo teórico-reflexivo que se apresenta com três eixos temáticos: i) orientações de enfermagem voltadas para prevenção de agravos urinários ii) orientações de enfermagem voltadas para prevenção de agravos intestinais; iii) trabalho de enfermagem em tempos de pandemia; Resultados: A análise da literatura demonstrou condições laborais indignas, caracterizadas pelo subdimensionamento de pessoal e escassez de materiais. Sobretudo, com a pandemia da COVID-19 constatou-se um desabastecimento dos Equipamentos de Proteção Individual. Assevera-se também que a paramentação e o trabalho intenso dificultam aos profissionais adotarem práticas que assegurem a saúde urinária e intestinal. É importante adotar práticas que possibilitem a saúde urinária: posição sentada para urinar, tempo apropriado para o completo esvaziamento vesical, facilidade de acesso sanitário durante o trabalho, adequada ingestão de líquidos, redução de substâncias irritantes vesicais como cafeína e cítricos. Assim como, elencam-se orientações para garantir a saúde intestinal: dieta com fibras; ingestão de líquidos; atividade física regular; uso de um suporte para melhorar a postura corporal no momento da evacuação, estabelecer uma rotina evacuatória. Conclusão: É relevante criar meios para socializar o conhecimento sobre promoção da saúde urinária e intestinal entre os profissionais de enfermagem, bem como estimular os gestores a viabilizar meios que garantam essa condição, melhorando o redimensionamento das equipes e objetivando a diminuição de complicações como constipação intestinal e incontinência urinária, dentre outros.
\end{abstract}

Palavras-chave: Enfermagem; COVID-19; Infecções por Coronavírus; Constipação intestinal; Incontinência urinária.

\begin{abstract}
Objective: To analyze the impact of the COVID-19 pandemic on the urinary and intestinal health of nursing professionals. Method: theoretical-reflective study with three thematic axes: i) nursing guidelines aimed at preventing urinary problems; ii) nursing guidelines aimed at preventing intestinal problems; iii) nursing work in pandemic time. Results: Unhealthy working conditions are observed in the health sector, characterized by undersized personnel and material shortages. Above all, with the COVID-19 pandemic, there was a shortage of Personal Protective Equipments. It is also asserted that vesting and intense work make it difficult for professionals to adopt practices that ensure urinary and intestinal health. It is important to adopt practices that enable urinary health: sitting position to urinate, appropriate time for complete bladder emptying, facility of toilet access during work, adequate fluid intake, reduction
\end{abstract}


of bladder irritating substances such as caffeine and citrus. As well, there are guidelines to guarantee intestinal health: fiber diet; fluid intake; regular physical activity; use of a support to improve body posture at the time of evacuation, establish an evacuation routine. Conclusion: It is relevant to create means to socialize the knowledge about the promotion of urinary and intestinal health of professionals, as well as to stimulate managers to make possible means that guarantee this condition, improving the resizing of the teams and aiming at reducing complications such as constipation and urinary incontinence, among others.

Keywords: Nursing; COVID-19; Coronavirus infections; Constipation; Urinary incontinence.

\begin{abstract}
Resumen
Objetivo: analizar el impacto de la pandemia de COVID-19 en la salud de la orina y el intestino de los profesionales de enfermería. Método: estudio teórico-reflexivo con tres ejes temáticos: i) el trabajo de enfermería en tiempos de pandemia; ii) pautas de enfermería destinadas a prevenir problemas urinários; iii) pautas de enfermería destinadas a prevenir problemas intestinales. Resultados: Se observan condiciones de trabajo indignas en el sector salud, caracterizadas por personal insuficiente y escasez de materiales. Sobre todo, con la pandemia de COVID-19, ha habido escasez de equipo de protección personal. También se afirma que el uso del equipos de protección personal (EPP) y el trabajo intenso dificultan que los profesionales adopten prácticas que aseguren la salud urinaria e intestinal. Es importante adoptar prácticas que permitan la salud urinaria: posición sentada para orinar, tiempo adecuado para el vaciado completo de la vejiga, facilidad de acceso sanitario durante el trabajo, ingesta adecuada de líquidos, reducción de sustancias irritantes de la vejiga como cafeína y cítricos. Además, existen pautas para garantizar la salud intestinal: dieta con fibra; ingesta de líquidos; actividad física regular; uso de un soporte para mejorar la postura corporal en el momento de la evacuación, establecer una rutina de evacuación. Conclusión: Es relevante crear medios para socializar el conocimiento sobre la promoción de la salud urinaria e intestinal de los profesionales, así como estimular a los gestores a hacer posibles los medios que garanticen esta condición, mejorando el redimensionamiento de los equipos y apuntando a reducir complicaciones como el estreñimiento y la incontinencia urinaria, entre otras.
\end{abstract}

Palabras clave: Enfermería; COVID-19; Infecciones por Coronavirus; Estreñimiento; Incontinencia urinaria.

\title{
1. Introdução
}

Os trabalhadores da saúde vendem sua força de trabalho para um determinado empregador, em condições laborais diversas a depender das características da organização nos quais estão inseridos, além de desenvolverem processos cujas metas se desejam alcançar, e para este fim se utilizam de instrumentos de atividades materiais e imateriais, os quais possibilitam a execução da tarefa laboral (Druck, 2016).

Neste sentido, com vistas à segurança na execução dos procedimentos para assistência à saúde, os Equipamentos de Proteção Individual (EPI’s) são produtos imprescindíveis, tanto entre os profissionais de saúde como entre pessoas nas comunidades, os quais incluem: luvas, máscaras cirúrgicas (ou padrão N95 ou FFP2), óculos, escudo facial ou equivalente, e aventais para procedimentos específicos, e o nível de assistência prestada por cada profissional irá definir a necessidade específica de cada EPI (OMS, 2020).

Em dezembro de 2019, a Organização Mundial de Saúde (OMS) declarou a ocorrência uma pandemia causada por um tipo de coronavírus (SARV-Cov-2) causador da doença COVID-19, o qual gerou uma preocupação internacional e impactou na disponibilidade dos EPI’́s. Sua transmissão se dá por meio de contato com as gotículas respiratórias de pessoas contaminadas através do espirro, tosse, catarro, objetos ou superfícies contaminadas (celulares, maçanetas, brinquedos, etc.) e toque através do aperto de mão (MS, 2020).

As dificuldades de abastecimento e logística dos EPI's estão relacionadas ao aumento de sua demanda e a limitação da capacidade de expansão de sua produção, sendo agravada pelo fato do seu uso indiscriminado e equivocado. Nesta perspectiva, é fundamental o conhecimento de suas especificidades e uso apropriado, como, por exemplo, a contraindicação do uso de máscaras acima de 4 horas, a depender de sua fabricação e composição, devido ao seu incômodo e riscos de lesão por dispositivos médicos o que pode impactar na assistência, na qualidade de vida e na autoestima dos profissionais, bem como apresentar-se como porta aberta para infecções (AMIB, 2020; Ramalho et al., 2020).

Neste contexto, discute-se como esse profissional que cuida diretamente dos pacientes infectados pela COVID-19 vem preservando sua saúde urinária e intestinal com base no que é recomendado pela literatura, tanto no que se refere à 
ingestão hídrica e alimentar, quanto em relação às condiçães de realização da micção e da evacuação durante seu turno de trabalho, pois alguns EPI's são de difícil desparamentação (Ramalho et al., 2020).

Assevera-se a isso, o medo de contaminação e o tempo demandado durante a troca dos EPI's, além da escassez destes produtos nas instituições de saúde, exigindo destes profissionais o controle de suas necessidades fisiológicas em prol do cuidado com os pacientes e a economia dos produtos, negligenciando sua saúde urinária e intestinal.

Corroborando com os achados, alguns ambientes de trabalho e suas demandas podem dificultar o acesso à água e, consequentemente, os profissionais diminuem sua frequência de uso do banheiro, além de propositalmente apresentarem uma baixa ingestão de líquidos com o objetivo de suprimir a vontade de urinar. Tais comportamentos podem causar desconforto e distensão vesical, além de aumentar o risco de infecções do trato urinário (Pierce et al., 2019).

De acordo com a Sociedade Internacional de Continência (ICS), o termo LUTS (Lower Urinary Tract Symptoms) refere-se a um grupo de sintomas relacionados ao armazenamento vesical, à micção e à pós-micção, os quais impactam negativamente na qualidade de vida dos indivíduos. Distúrbios emocionais como depressão e ansiedade, atividade sexual reduzida, interferência em atividades sociais relacionados à vergonha, constrangimento, frustração são alguns exemplos dos efeitos dos LUTS, e que são vivenciados por mais de 50\% da população feminina. Neste contexto, existe uma maior vulnerabilidade de LUTS em enfermeiras, onde $65 \%$ relataram pelo menos um tipo de sintoma de LUTS, os quais são atribuídos a fatores como sobrecarga e altos níveis de tensão no trabalho (Wan et al., 2017; Angelo et al., 2020).

Outra definição importante para avaliação da saúde urinária são as ações voluntárias relacionadas ao esvaziamento fisiológico da bexiga denominadas comportamentos sanitários, as quais incluem selecionar o local da micção, tempo da atividade de esvaziamento vesical, posição adotada durante o ato miccional e o estilo miccional (Angelini et al., 2019).

O comportamento sanitário apresenta conexão com os LUTS e as disfunções miccionais, podendo incluir: micção prematura (urinar sem a necessidade ou urgência), esforço ou aceleração durante o esvaziamento vesical, bem como seu retardamento quando não houver fácil acesso ao sanitário (Angelini et al., 2019).

Ainda nesse contexto, faz-se necessári9o destacar a incontinência urinária que é a perda de urina de caráter involuntário, condição em que muitos indivíduos convivem com esse problema acreditando ser normal e sentem-se constrangidos de comentar com familiares e amigos, ou ainda de buscar ajuda profissional. Contudo, existem repercussões econômicas, sociais, psicológicas e de saúde que este acometimento gera (Abrams et al., 2017; Wu et al.,2019).

Paralelamente, o hábito intestinal é caracterizado não só pela frequência evacuatória como também pela consistência das fezes e pela demanda de esforço para evacuar. O padrão intestinal considerado normal está relacionado à presença de uma evacuação a cada três dias ou três evacuações diárias, assim como a presença de fezes macias ou pastosas e a inexistência de esforço evacuatório (Shimidt et al., 2016).

Uma das principais complicações do hábito intestinal prejudicado é a constipação intestinal (CI), a qual é definida pela presença de dois dos seguintes sintomas presentes durante três meses nos últimos seis meses antes do diagnóstico: menos de três evacuações por semana, presença de fezes endurecidas e a percepção de evacuação incompleta, a sensação de obstrução ou interrupção da evacuação, manobras manuais para facilitar a evacuação (Sobrado et al., 2018).

Considerando a problemática contextualizada, julgou-se relevante elaborar o presente estudo que tem como objetivo: analisar o impacto da pandemia da COVID-19 na promoção da saúde urinária e intestinal dos profissionais de enfermagem.

A contribuição deste estudo visa a promoção de orientações voltadas para a saúde urinária e intestinal dos profissionais de enfermagem com vistas a diminuição de agravos na sua saúde biopsicossocial, bem como, para sua qualidade de vida. Sobretudo, considerando as dificuldades impostas pela assistência a pessoas com COVID-19, que demandam a paramentação e desparamentação frequente, requerendo elevada carga de trabalho e ritmo laboral intenso desfavorecendo, muitas vezes, tais práticas de saúde. 


\section{Metodologia}

Este é um estudo teórico-reflexivo sobre o impacto da pandemia da COVID-19 na saúde urinária dos profissionais de enfermagem, o qual se caracteriza por possibilitar descrições e interpretações sobre determinado fenômeno inquietante e efetuar análises fundamentando-se na formulação discursiva que teve como base a leitura crítica de publicações (nacionais e internacionais) sobre literatura pertinente à área da saúde urinária e intestinal, biossegurança e trabalho de enfermagem. As análises de referenciais teóricos, publicações de organizações, órgãos certificadores e artigos científicos, disponibilizados na Biblioteca Virtual em Saúde (BVS), Public/Publisher MEDLINE (PubMed) e sites especializados, subsidiaram a discussão acerca da temática.

Tal estudo permeia as pesquisas de abordagem qualitativa no que se refere a fundamentação na descrição e análise de constructos teóricos apreendidos por via de levantamento bibliográfico o que promove um aprofundamento na compreensão do objeto de estudo (Minayo, 2006).

Em seguida, após a organização e ordenação lógica das categorias (Pereira et al, 2018), foi elaborado o texto teórico reflexivo cuja discussão foi norteada a partir de três eixos temáticos: i) orientações de enfermagem voltadas para prevenção de agravos urinários ii) orientações de enfermagem voltadas para prevenção de agravos intestinais. iii) trabalho de enfermagem em tempos de pandemia.

\section{Resultados e Discussão}

\section{Orientações de enfermagem voltadas para prevenção de agravos urinários.}

Inúmeros fatores podem estar relacionados à IU como idade, raça, educação, composição corporal, estado civil, horas e experiência de trabalho, ingestão de líquidos, infecção urinária prévia e constipação crônica (Assis, et al., 2020).

Dentre os tipos de IU, três merecem destaque: 1) de esforço, quando existe escape de urina ao tossir, realizar atividades físicas; 2) de urgência, também conhecida como hiper-reflexia detrusora idiopática, a qual trata-se de perda involuntária de urina durante uma necessidade urgente de urinar, e a 3) mista, que compreende os dois tipos (de esforço e de urgência). $\mathrm{O}$ tratamento não farmacológico e não cirúrgico consiste em mudanças comportamentais, biofeedback e treinamento dos músculos do assoalho pélvico (Assis, et al., 2020; Valença et al., 2016).

Uma posição relaxada e o tempo apropriado para o completo esvaziamento vesical são necessários para otimizar a saúde da bexiga. Porém, o uso de sanitários públicos pode demandar padrões de comportamentos alterados devido à preocupação de algumas mulheres com a sua limpeza, as quais tendem a evitá-los favorecendo o desenvolvimento de LUTS. Existem relatos de atraso da micção até chegarem em casa ou também de urinar antes de sair de casa, por exemplo (Angelini et al.,2019).

Pesquisadores ainda referem que aliado a estes comportamentos, $85 \%$ das mulheres também adotam posição típica nos banheiros públicos a qual pode vir a dificultar o relaxamento do assoalho pélvico, que é descrita como o agachamento sobre o assento sanitário sem manter contato com este; também relatam o retardamento do ato de urinar; somando-se o fato da manobra de Valsalva ser prejudicial ao colo vesical em mulheres, o que é preditivo em LUTS (Angelini et al.,2019; Arnetz, et al., 2020; Abrams, et al., 2017).

Entre as enfermeiras, a carga laboral excessiva e a tensão do ambiente de trabalho são situações que influenciam diretamente no aumento dos comportamentos sanitários anormais. Salienta-se que esses comportamentos estão relacionados ao retardamento da necessidade da micção devido a pausas inadequadas durante o plantão, um limitado número de banheiros disponíveis e saneamento deficiente. Tal situação promove o desconforto vesical, o que pode acarretar a distensão vesical, sensação de bexiga alterada, e aumento do risco de infecções do trato urinário (Dongiuan, et al., 2019). 
Estudo realizado para correlacionar os comportamentos sanitários e a presença de LUTS entre enfermeiras, identificou que uma em cada quatro enfermeiras referem ter acesso restrito ao sanitário durante o trabalho, bem como a maioria das entrevistadas relatam o adiamento da vontade de urinar. A ingestão de líquidos limitada também foi descrita como uma necessidade para retardar a micção, e quase metade registrou impacto significativo de LUTS pela necessidade de enfrentamento de tais sintomas durante sua jornada de trabalho. Algumas enfermeiras relataram também que o fato de retardar a necessidade de urinar influenciou na sua habilidade de concentração durante o trabalho (Wan et al., 2017).

Vale salientar que o referido estudo foi realizado sem o contexto da pandemia do COVID-19. Porém, ao considerar o momento pandêmico, a problemática em questão pode ser agravada por conta da necessidade de paramentação mais rigorosa e que demanda mais tempo da equipe de saúde para sua realização, bem como para se desparamentar. Além disso, muitos pacientes necessitam de cuidados intensivos, procedimentos mais complexos, e consequentemente demandam elevada atenção e cuidados da equipe de saúde.

Considerando ainda que os profissionais de enfermagem são, geralmente, mais propensos a relatar ansiedade, depressão e sintomas físicos como dores nas costas, e tais relatos impactam na presença de LUTS, outros fatores podem agravar ainda mais a saúde biopsicossocial dos profissionais de saúde, como a insegurança e o medo provocados pela pandemia do COVID-19. Acrescentam-se ainda os dados epidemiológicos alarmantes, os riscos de aquisição e transmissão da doença, a perda de pacientes, as jornadas de trabalho exaustivas, o uso frequente de EPI's e os riscos de lesão na pele que também são motivos para agravos à saúde (Wan et al., 2017; Arnetz et al., 2020).

Corroborando com os achados, estudo demostrou que $70 \%$ dos profissionais de saúde, na linha de frente para o combate do COVID-19 em Wuhan, apresentaram níveis extremos de estresse, com sintomas de depressão (50\%), ansiedade (44\%) e insônia (34\%). Com grandes níveis de estresse ocupacional, os enfermeiros tendem a adotar comportamentos sanitários não saudáveis para o esvaziamento vesical e isso aumenta as chances de desenvolver problemas urinários como a bexiga hiperativa (Dongiuan et al., 2019; Lai et al., 2020).

O nível de estresse também impacta na frequência urinária pela correlação do sistema urinário com o sistema límbico localizado no sistema nervoso central. Esse estímulo promove uma tensão muscular que aumenta o desejo da micção, mesmo com pouca quantidade de urina (Assis et al., 2020).

Cabe salientar que a constipação intestinal crônica contribui para o enfraquecimento do assoalho pélvico e predispõe a incontinência urinária por demandar força para evacuar inúmeras vezes, e o esforço prejudica as fibras musculares que dão suporte à região pélvica (Assis et al., 2020).

Um consenso de experts em práticas de saúde urinária inclui a recomendação da adequada ingestão de líquidos (25-30 $\mathrm{ml}$ por quilo de peso corporal por dia), pois a inadequada ingestão hídrica está associada às infecções do trato urinário, constipação e pode alterar, negativamente, a cognição. Em casos mais graves, a desidratação crônica pode acarretar vários problemas como litíase renal, câncer de bexiga e doença cardiovascular (Pierce et al., 2019).

Além disso, a baixa ingestão hídrica tem como consequência a maior concentração urinária, o que promove aumento da sensibilidade e irritação vesical, e estes fatores são contribuintes para a incontinência urinaria de urgência (Assis et al., 2020).

Como importante comportamento sanitário, o ato de sentar para urinar é de extrema relevância, pois promove o relaxamento dos músculos do assoalho pélvico e esvaziamento vesical completo, evitando a incontinência urinária e as infecções do trato urinário. Sugere-se um planejamento da frequência miccional de quatro a sete vezes por dia, não sendo recomendado ultrapassar quatro horas de intervalo entre as micções (Pierce et al., 2019; Lai et al., 2019). 
Outras recomendações para saúde urinária incluem reduzir substâncias irritantes vesicais como cafeína e cítricos, melhorar a função intestinal no intuito de evitar a constipação crônica e realização precoce e constante de exercícios para fortalecimento do assoalho pélvico (Assis et al., 2020).

Portanto, a necessidade de facilitar o acesso ao sanitário durante o trabalho é uma questão importante para os profissionais de enfermagem, pois o atraso no ato da micção e a ingestão inadequada de líquidos, práticas corriqueiras destes profissionais, podem impactar, negativamente, na produtividade e compromisso com o cuidado clínico, além de predispor aos sintomas urinários e desenvolvimento de sérias condições de saúde, gerando absenteísmo entre esses trabalhadores.

Além disso, os gestores de saúde necessitam dispor de recursos humanos e físicos suficientes de forma a permitir o tempo de troca de EPI’s, além do uso adequado da toalete, de forma a não prejudicar a saúde urinária desses profissionais.

\section{Orientaçães de enfermagem voltadas para prevenção de agravos intestinais}

A constipação intestinal não apresenta um fator isolado, possibilitando associações entre alterações sistêmicas ou neurológicas e a utilização de medicamentos e dietas inapropriadas com consumo de fibras diminuído, idade avançada, sexo feminino, baixos nível socioeconômico e escolaridade, distúrbios endocrinometabólicos, ausência de hábitos de exercícios físicos, distúrbios psiquiátricos e causas idiopáticas. Em contrapartida, após investigação rigorosa pode ser verificado como estágio inicial de tumores colorretais (Jaques et al., 2020)

A constipação intestinal (CI) atinge em sua maioria os adultos, dentre os quais existe uma maior predominância nos idosos devido às alterações fisiológicas do envelhecimento como mobilidade reduzida, condições de saúde alterada, uso de medicamentos etc. Nas mulheres a CI está relacionada à fatores como gravidez, tipo de parto, alterações hormonais específicas do sexo, e histórias de abuso sexual, físico e emocional. Apesar de não representear uma ameaça à vida, ela apresenta um grande impacto na qualidade de vida principalmente na sua forma crônica (Schmidt et al., 2015; Sobrado et al., 2018).

Além da análise do hábito intestinal, o tipo de fezes contribui para a investigação de doenças relacionadas ao trânsito intestinal, como a síndrome do intestino irritável, a própria constipação intestinal e a incontinência anal, por exemplo. Para este fim, a escala de Bristol foi um instrumento desenvolvido para que a pessoa consiga identificar o tipo de fezes que mais se assemelha ao seu, pois apresenta gráficos com imagens de sete diferentes tipos de forma e consistência fecal, sendo cada uma relacionada também à sua descrição (Martinez e Azevedo, 2012).

O tipos de fezes descritos no instrumento são: tipo 1 (pequenas bolinhas duras, separadas como coquinhos - difícil para ser eliminada), tipo 2 (formato de linguiça encaroçada, com pequenas bolinhas agrupadas), tipo 3 (formato de linguiça com rachaduras na superfície), tipo 4 (alongada com formato de salsicha ou cobra, lisa e macia), cuja literatura aponta como sendo as mais adequadas, tipo 5 (pedaços maiores e separados com bordas bem definidas - fáceis de serem eliminadas, tipo 6 (massa pastosa e fofa, com bordas irregulares), e o tipo 7 (totalmente líquidas, sem pedaços sólidos). (Jaques et al., 2020; Martinez e Azevedo, 2012).

Cabe salientar que a constipação intestinal crônica tem como consequências complicações clínicas severas como fecaloma, pseudodiarreia, volvo, além de ser um fator de risco para o desenvolvimento da incontinência urinária e baixa qualidade de vida (Assis et al., 2020; Schmidt et al., 2015; Wu et al., 2019).

Destarte, assevera-se que tal alteração do hábito intestinal nos profissionais tem como fator agravante as longas horas de plantão, em ambientes fechados, com atividade laboral que demanda a execução de procedimentos demorados. Outrossim, destaca-se a seriedade e responsabilidade de cuidar de pacientes cuja doença ainda é desconhecida a todos, a qual evolui com manifestações insólitas, cujo alto volume de procedimentos cansam e elevam o nível de estresse da equipe de enfermagem. Dessa forma, a ampla divulgação das orientações de guidelines nacionais e internacionais se faz necessária, com o intuito de promover os cuidados com a saúde intestinal e a prevenção de agravos à saúde. 
Neste sentido, para a obtenção da saúde intestinal, as orientações são: dieta com grande suporte em fibras; ingestão de líquidos se não houver doença que contraindique o aumento da ingestão hídrica; atividade física regular; fazer uso de um suporte para melhorar a postura corporal no momento da evacuação, estabelecer uma rotina evacuatória sentando-se no vaso sanitário preferencialmente no mesmo horário após as refeições; realização da manobra de Valsalva, que consiste em inspirar profundamente e forçar os músculos do abdômen e diafragma para baixo; além da realização por vinte minutos de massagens abdominais no sentido do cólon da direita para esquerda e de cima para baixo (Jaques et al., 2020; Schmidt et al., 2015

É importante ressaltar que a ingestão de líquidos, incluindo sucos e água, deve ser de pelo menos oito copos/dia, pois a hidratação adequada altera a consistência e o peso das fezes, aumenta o número de reflexos gastrocólicos e contribui para a lubrificação intestinal. Além disso, as fibras alimentares presentes nas frutas e verduras são componentes não digeridos pelas enzimas digestivas do trato gastrintestinal dos seres humanos, dividindo-se em solúveis que colaboram para a maciez das fezes e insolúveis as quais aumentam o volume fecal, estimulando as ondas peristálticas e o esvaziamento do cólon (Sobrado et al., 2018).

Diante deste contexto, entende-se que medidas simples tanto comportamentais quanto alimentares podem ser incorporadas durante as atividades laborais das equipes de saúde de maneira que possa sem implementada a curto, médio e longo prazo, contribuindo para a qualidade de vida dos profissionais de saúde.

\section{Trabalho de enfermagem em tempos de pandemia}

$\mathrm{O}$ trabalho vem assumindo, cada vez mais, centralidade na vida das pessoas, o que traz consequências paradoxais para a integridade física, psíquica e social dos trabalhadores, podendo repercutir em prazer ou em sofrimento, em satisfação ou em insatisfação e, sobretudo, em saúde ou em doença (Dejours, 2012).

O objeto de trabalho da enfermagem é o cuidado, que é desenvolvido na perspectiva tanto de prevenção de doenças, como na promoção e recuperação da saúde, bem como de reabilitação. Dessa forma, este profissional aplica seu conhecimento específico, em uma dada estrutura organizacional, com instrumentos apropriados, ou seja, métodos, materiais e equipamentos, os quais proporcionam o cuidado para assistir pessoas necessitadas ou em situação de vulnerabilidade (Gonçalves, 2015).

Sendo assim, além de contar com uma força de trabalho com formação e qualificação específicas para prestar o cuidado, é preciso que a organização laboral possibilite meios para que a produção se desenvolva a contento, ou seja, com um quantitativo adequado dos recursos humanos, qualidade e quantidade de insumos e equipamentos apropriados para a especificidade da atividade a ser executada, espaço físico condizente ao bom desenvolvimento do processo laboral, comunicação eficiente e efetiva, bem como o incentivo e viabilidade ao trabalho em equipe (Dias et al., 2019).

No entanto, contraditoriamente, verifica-se no sistema de saúde brasileiro condições indignas de trabalho, caracterizadas por salários aviltantes, subdimensionamento de pessoal, inadequada qualificação dos recursos humanos, falta de estrutura física para o desenvolvimento do cuidado, não observância de manutenção dos equipamentos e escassez qualitativa e quantitativa de materiais. Nesta perspectiva, destaca-se a carência de equipamentos de proteção individual, os quais são primordiais para salvaguardar a saúde dos profissionais e protegê-los dos riscos ocupacionais (Dias et al., 2019).

Sobretudo, com a pandemia da COVID-19 constatou-se um desabastecimento dos EPI's devido a alguns fatores como, por exemplo, um planejamento equivocado das instâncias governamentais e das gerências dos serviços a fim de suprir as necessidades dos trabalhadores da saúde no exercício das suas funções; a aquisição desses equipamentos pela população em geral levando à carência destes no mercado; o uso não racional dos EPI’s pelos profissionais, entre outras situações. Verific ouse também equipamentos de proteção individual com qualidade inadequada e/ou ergonomicamente inapropriados (Souza et al., 2020). 
Salienta-se que um dos desdobramentos da situação de escassez de EPI é o seu uso pelos trabalhadores por tempo maior que o recomendado pelo fabricante, por receio da não reposição ou pela sua própria carência, o que gera dúvidas em relação a capacidade de proteção contra os riscos ocupacionais. Destaca-se ainda a ocorrência de lesões de pele decorrentes dos dispositivos médicos, especificamente pelos EPI (Carvalho et al., 2019).

Outra questão que necessita de reflexão é a alta demanda de trabalho e o ritmo laboral intenso originado pela carência de pessoal e pela complexidade e volume dos pacientes internados pela COVID-19. Tal intensificação do ritmo laboral dificulta que os profissionais tenham pausas durante o turno de trabalho para se alimentarem, hidratarem-se e irem ao banheiro, o que provoca problemas de saúde como déficits alimentares, alterações hidroeletrolíticas, infecção urinária, constipação, entre outras desordens patológicas.

\section{Considerações Finais}

Este estudo permitiu reflexões acerca da necessidade de promover a conscientização dos profissionais de saúde e gestores sobre a temática da saúde urinária e intestinal, sobretudo na perspectiva da prevenção de agravos. Ademais, tornou-se mais preocupante com o advento da pandemia da COVID-19, que potencializou os fatores desencadeantes de doenças do trato urinário e intestinal.

Nesse sentido, o uso de EPI's por longos períodos, a difícil desparamentação, pacientes complexos que carecem de atenção e de procedimentos constantes, resultando na alta demanda laboral, banheiros de difícil acesso e com higienização duvidosa, são aspectos que têm promovido mais impactos negativos na saúde urinária e intestinal dos profissionais de enfermagem.

Assim, faz-se relevante desenvolver estratégias para socializar o conhecimento de como promover a saúde focando nessa perspectiva e, ao mesmo tempo, sensibilizar gestores para viabilizarem meios que possibilitem adesão dos profissionais de enfermagem aos comportamentos sanitários adequados à preservação da saúde urinária e intestinal.

Entende-se que a limitação deste manuscrito está em ser de reflexão, o qual fica restrito ao que já foi publicado sobre a temática. No entanto, considera-se que é relevante no sentido de compilar dados sobre o foco do estudo e, assim, socializar o conhecimento adicionado à experiência dos pesquisadores. Sobretudo, a presente pesquisa pode servir de alerta aos profissionais e gestores sobre a importância e os desafios para preservação da saúde urinária e intestinal.

Por fim, faz-se relevante o desenvolvimento de pesquisas que descrevam as condições de saúde urinária e intestinal dos profissionais de enfermagem, bem como suas complicações, além da construção de instrumentos que viabilizem a identificação de fatores que possam influenciar direta e indiretamente a saúde destes profissionais.

\section{Referências}

Abrams, P., Cardozo, L., Wagg, A., \& Wein, A. (2017) Incontinence. (6a ed.), International Continence Society.

Angelini, K. J., Newman, D. K., \& Palmer, M. H. (2019) Psychometric Evaluation of the Toileting Behaviors: Women's Elimination Behaviors Scale in a Sample of College Women Female Pelvic Medicine \& Reconstructive. 26(4) - p 270-275 10.1097/SPV.0000000000000711

Angelo, P. H., Queiroz, N. A., Leitão, A. C. R., Marini, G., \& Micussi, M. T. (2020) Validation of the international consultation on incontinence modular questionnaire - female lower urinary tract symptoms (ICIQ-FLUTS) into brazilian Portuguese Int Braz J Urol. 46(1) 10.1590/S1677-5538.IBJU.2019.0234

Arnetz, J. E., Goetz, C. M., Sudan, S., Arble, E., Janisse, J., \& Arnetz, B. B. (2020) Personal Protective Equipment and Mental Health Symptoms Among Nurses During the COVID-19 Pandemic. J Occup Environ Med; 62(11): 892-897 10.1097/JOM.0000000000001999.

Assis, G. M., Goulart, M. L., Nunes, A. C. S., \& Oliveira, F. F. (2020) Prevenindo e tratando a incontinência urinária feminina - Taubaté: Casa Cultura, 28 pág. / 210x297mm

Associação de Medicina Intensiva Brasileira (AMIB). (2020) Na UTI, a segurança da equipe é fundamental! ttps://www.amib.org.br/fileadmin/user_upload/amib/2020/marco/07/COVID-19_seguranca_equipev14032020_18h16.pdf 
Bharucha, A. E., \& Wald, A. (2019) Chronic Constipation Mayo Clin Proc.; Mayo Foundation for Medical Education and Research 94(11):2340-2357 10.1016/j.mayocp.2019.01.031

Carvalho, E. C., Souza, P. H. D. O., Dantas, O., Varella, T. C. M. M. L., Souza, N. V. D. O., Farias, S. N. P., \& Soares, S. S. S. Pandemia da COVID-19 e a judicialização da saúde: estudo de caso explicativo.(2020) Rev. Latino-Am. Enfermagem [Internet]. 28. Article e3354 10.1590/1518-8345.4584.3354.

Dejours, C. (2012) Psicodinâmica do trabalho: contribuições da Escola Dejouriana à análise da relação prazer, sofrimento e trabalho. Atlas.

Dias, M. O., Souza, N. V. D. O., Penna, L. H. G., \& Gallasch, C. H. (2019) Perception of nursing leadership on the fight against the precariousness of working conditions. Rev Esc Enferm USP Article e03492. 10.1590/S1980-220X2018025503492.

Dongjuan, X. U., Shanshan, Z. H. U., Hui, L. I., Jie, G. A. O., Huanyu, M. O. U., \& Kefang, W. (2019) Relationships among occupational stress, toileting behaviors, and overactive bladder in nurses: A multiple mediator model. J Adv Nurs .75(6):1263-1271. 10.1111/jan.13940.

Druck, G. A. (2016) Terceirização na saúde pública: formas diversas de precarização do trabalho. Trab. Educ. Saúde, Rio de Janeiro, 14(1). <http://www.scielo.br/scielo.php?script=sci_arttext\&pid=S1981-77462016000400015\&lng=en\&nrm=iso>.

Gonçalves, F. G. A., Souza, N. V. D., Zeitoune, R. C. G., Adame, G. F. P. L., \& Nascimento, S. M. P. (2015) Impactos do neoliberalismo no trabalho hospitalar de enfermagem, Texto Contexto Enferm., Florianópolis, 24(3), p.646-653. <http://www.scielo.br/pdf/tce/v24n3/pt_0104-0707-tce-24-0300646.pdf $>$.

Jaques, R. M. P. L., Costa, E. C. L., Assis, G. M., Oliveira, A. C., Benício, C. D. A. V., \& Bezerra, S. M. G. (2020) Perfil sociodemográfico e de eliminações intestinais em mulheres atendidas em um serviço de saúde. ESTIMA, Braz. J. Enterostomal Ther., 18: e0820 10.30886/estima.v18.830_PT

Lai, J., Ma, S., Wang, Y., Cai, Z., Hu, J., \& Wei, N. (2020) Factors Associated With Mental Health Outcomes Among Health Care Workers Exposed to Coronavirus Disease 2019. JAMA Netw Open. Mar 2;3(3) Article: e203976. 10.1001/jamanetworkopen.2020.3976.

Martinez, A. P., \& Azevedo, G. R. (2012) Tradução, adaptação cultural e validação da Bristol Stool Form Scale para a população brasileira Rev. Latino-Am. Enfermagem [Internet]. 20(3):[7 telas]. www.eerp.usp.br/rlae

Minayo, M. C. S. (2006) O desafio do conhecimento: pesquisa qualitativa em saúde. (9a ed.), EDUC

Ministério da saúde (MS). (2020) Manejo clínico e tratamento da COVID-19. <https://coronavirus.saude.gov.br/sobre-a-doenca\#transmissao) >

Organização Mundial da Saúde (OMS). (2020) Rational use of personal protective equipment for coronavirus disease 2019 (COVID-19): Interim guidance. Genebra: WHO. Retrieved from< https://apps.who.int/iris/bitstream/>

Pereira, A, S.; Shitsuka, D. M.; Parreira, F. J.; \& Shitsuka, R. (2018) Metodologia da pesquisa científica. UFSM. https://repositorio.ufsm.br/bitstream/handle/1/15824/Lic_Computacao_Metodologia-Pesquisa-Cientifica.pdf?sequence=1

Pierce, H. M., Perry, L., Gallagher, R., \& Chiarelli, P. (2019) Delaying voiding, limiting fluids, urinary symptoms, and work productivity: A survey of female nurses and midwives. J Adv Nurs.; 75:2579-2590 10.1111/jan.14128Aa

Ramalho, A. O., Freitas, P. S. S. \& Nogueira, P. C. (2020) Lesão por pressão relacionada a dispositivo médico nos profissionais de saúde em época de pandemia. ESTIMA, Braz. J. Enterostomal Ther., 18: Article e0120 10.30886/estima.v18.867_PT

Schmidt, F. M. Q., Santos, V. L. C. G., Domansky, R. C., Barros, E., Bandeira, M. A., \& Tenório, M. A. M. (2015) Prevalência de constipação intestinal autorreferida em adultos da população geral. Rev Esc Enferm USP.;49(3):443-52.1 10.1590/S0080-623420150000300012

Shimidt, F. M. Q. Azevedo, G. R., Silveira, N. I., Paula, M. A. B., Ricarte, M. C., \& Jorge, S. A. (2016) Intervenções nas Áreas de Abrangência da Estomaterapia Instituto Santa Teresa / Centro Universitário Teresa D'Ávila Lorena, São Paulo http://www.sobest.org.br/arquivos/PDF_INTERVENCOES.pdf

Sobrado, C. W., Corrêa, N. I. J. F., Pinto, R. A., Sobrado, L. F., Nahas, S. C., \& Cecconello, I. (2018) Diagnosis and treatment of constipation a clinical update based on the Rome IV criteria. J Coloproctol. 38(2):137-44. 10.1016/j

Souza, N. V. D. O., Dias, M., Carvalho, E. C., Varella, T. C. M. M. L, Lima, L. S. C., \& Soares, S. (2020) Risco de uberização do trabalho de enfermagem em tempos de pandemia da Covid-19: relato de experiência. Research, Society and Development, 9(10), Article e:7629109060. <https://rsdjournal.org/index.php/rsd/article/view/9060>.

Valença, M. P., Albuquerque, A. F. L. L., Rocha, G. M. S., \& Aguiar, A. P. D. (2016) Cuidados de Enfermagem na Incontinência Urinária: um Estudo de Revisão Integrativa ESTIMA, Braz. J. Enterostomal Ther., 14(01), p. 43-49 10.5327/Z1806-3144201600010007

Wan, X., Wuc, C., Xua, D., Huanga, L., \& Wnaga, K. (2017) Toileting behaviours and lower urinary tract symptoms among female nurses: A cross-sectional questionnaire survey. International Journal of Nursing Studies. 65: 1-7.

Wu, C., Xue, K., \& Palmer, M. H. (2019) Toileting Behaviors Related to Urination in Women: A Scoping Review. Int J Environ Res Public Health. 16(20); 4000. 10.3390/ijerph16204000. 